6. Martino J, Sebert S, Segura MT, et al. Maternal Body Weight and Gestational Diabetes Differentially Influence Placental and Pregnancy Outcomes. J Clin Endocrinol Metab. 2016;101(1):59-68. doi:10.1210/jc.2015-2590

7. Torloni MR, Betrán AP, Horta $B L$, et al. Prepregnancy BMI and the risk of gestational diabetes: a systematic review of the literature with meta-analysis. Obes Rev. 2009;10(2):194-203. doi:10.1111/j.1467-789X.2008.00541.x
8. Brunner $S$, Stecher $L$, Ziebarth $S$, et al Excessive gestational weight gain prior to glucose screening and the risk of gestational diabetes: a meta-analysis. Diabetologia. 2015;58(10):22292237. doi:10.1007/s00125-015-3686-5

9. Vũ Thanh Vân. Môt Số Nhân Xét về Bênh Đái Tháo Đường ở Phụ Nữ Có Thải ở Bệnh Viện Phụ Sản Trung Ương Năm 2010- 2011. Luân văn tốt nghiệp Bác sỹ Chuyên khoa 2, Trường Đại Học Y Hầ Nội.; 2012.

\title{
SƯ⿱ TĂNG TRƯỞNG ĐẦU MẶT Ở TRẺ EM NGƯỜI KINH TỪ 7 ĐẾN 9 TUỔI BẰNG PHƯƠ'NG PHÁP ĐO TRÊN ẢNH CHUẨN HÓA THẲNG VÀ NGHIÊNG
}

\author{
Trương Đình Khởi ${ }^{1}$, Lương Ngọc Khuê ${ }^{2}$, Đào Thị Dung ${ }^{3}$,
} Trần Văn Tiến ${ }^{2}$, Hà Ngọc Chiều ${ }^{1}$

\section{TÓM TẮT}

Mục tiêu: Phân tích sự tăng trưởng đâu mặt ở trẻ người Kinh 7 -9 tuổi bằng phương pháp đo trền ảnh chuẩn hóa thẳng và nghiêng. Đối tượng và phương pháp nghiên cứu: Nghiên cứu dọc trên 206 trẻ $7-9$ tuổi người Kinh tại trường Tiểu học Liên Ninh, Thanh trì, Hà Nội bằng phương pháp đo trên ảnh chuẩn hóa thẳng và nghiêng. Kết quá: Các kích thước chiêuu rộng mặt, chiều rộng miệng, chiều rộng hàm dưới, chiều cao tầng mặt trên ở nam lớn hơn ở nữ, không có sự khác biệt có ý nghĩa thống kê giữa ba nhóm khớp cắn theo phân loại Angle, trừ chiều rộng mũi, chiều cao tâng mặt giữa, chiều cao tầng mặt dưới ở loại khớp cắn loại III lớn hơn loại I,II Angle. Các kích thước đầu mặt tăng trưởng diến ra liên tục theo tuổi. Kết luâan: Các kích thước vùng đâu mặt ở nam lớn hơn ở nữ, tẳng trưởng ở nữ có xu hướng sớm hơn ở nam.

Tư khóa: chiều rộng mặt, chiêuu rộng mũi, tăng trưởng đâuu mặt

\section{SUMMARY \\ CRANIOFACIAL GROWTH IN KINH ETHNIC CHILDREN FROM 7 TO 9 YEARS OF AGE IN DIGITAL PHOTOGRAPH - A LONGITUDINAL STUDY}

Objectives: To analysis facial measurements and craniofacial growth in standardized photograph in King ethnic children from 7 to 9 years old. Subjects and methods: A longitudinal study of 206 children (104 males, 102 females) from 7 to 9 years old in Lien Ninh primary school, Thanhtri, Hanoi by measuring in standardized photograph. Results: Average of facial dimensions (facial width, width of mouth, mandibular

${ }^{1}$ Viện Đào tạo Răng Hàm Mặt- Đại học Y Hà Nội 2Cục Khám chữa bệnh và Cục Y tế dự phòng-Bộ y Tế ${ }^{3}$ Khoa Y Dước-Đại học Quốc Gia Hà Nội

Chịu trách nhiệm chính: Trương Đình Khởi

Email: Bskhoirhm@gmail.com

Ngày nhận bài: 5.3.2021

Ngày phản biên khoa hoc: 27.4 .2021

Ngày duyệt bài: 10.5.2021 width, superior height) in male were larger than in female there was not statistical difference among three groups. Conclusion: Most of craniofacial dimensions in male was larger than in female, facial growth in female was significantly sooner than in male.

Keywords: Facial width, nasal width, craniofacial growth

\section{I. ĐĂT VẤN ĐỀ}

Các đặc điểm nhân trắc khuôn mặt là yếu tố quan trọng để nhận dạng của một con người. Các đăc điểm hình thái đâu măt thể hiên rõ nét sư khác biêt trong dân tộc người này so với dân tộc khác. Có nhiều phương pháp đang được sử dụng hiện nay để đánh giá các đặc điểm nhân trắc khuôn mặt, một trong số đó là phương pháp đo đac được tiến hành trên ảnh chụp chuẩn hóa thẳng và nghiêng.

Trên thế giới đã có nhiêu nghiên cứu về hình thái đâu mặt, từ thời kỳ Phục hưng đã có phân loại hình thái khuôn mắt của Leonardo De Vinci (1452-1519), Michelangelo (1475-1564), Albrecht Durer (1471-1528) và Francis Galton (1822-1911) [1]. Nghiên cưu của J.Leon Williams (1920) đưa ra bốn loai hình thái đâu măt bao gôm: mặt tròn, mặt thuôn nhọn, mặt oval và mặt vuông, phân loại này vẫn thường được dùng cho đến ngày nay, nhưng chỉ dựa vào định tính [2]. Nghiên cứu của Celebic.A, Jerolimov.V (2001) chỉ ra phân loại hình thái đâu mặt dựa trên kích thước chiều rộng thái dương, chiểu rộng mă̆t và chiều rộng hàm dưới, đưa ra ba loại hình thái mắt chỉ dựa vào định lượng: Mặt oval, mặt vuông và mặt thuôn nhọn [3].

Tại Việt Nam, đã có một số nghiên cứu về hình thái đầu mặt như nghiên cứu của Lê Đức Lánh (2007) [4], Võ Trương Như Ngọc (2010) [5], Trương Hoàng Lệ Thưy, Nguyển Thị Kim Anh (2012) [6], Trân Tuấn Anh (2016) [7], tuy 
nhiên, chưa có nghiên cứu hình thái đầu mă̆t ở trẻ em 7 tuổi bằng phương pháp đo trên ảnh chuẩn hóa thẳng và nghiêng. Phương pháp đo trên ảnh chuẩn hóa thằng và nghiêng cho phép nghiên cứu được một số đăc điểm đầu măt, dễ dàng lưu trữ và trao đổi, có thể thực hiện với những dụng cụ, trang thiết bị nhỏ gọn. Chính vì vậy, chúng tôi tiến hành nghiên cứu đề tài "Sự tăng trưởng đầu măt ở trẻ em người Kinh từ 7 đến 9 tuổi bằng phương pháp đo trên ảnh chuẩn hóa thẳng và nghiêng" với mục tiêu: Xác định một số kích thước vưng đầu mặt và mô tả sự tăng trưởng đầu măt ở trẻ em người Kinh từ 7 đên 9 tuôi bằng phương pháp đo trên ảnh chuẩn hóa thẳng và nghiêng.

\section{II. ĐỐI TƯƠ'NG VÀ PHƯƠNG PHÁP NGHIÊN CỨU}

2.1 Đối tượng nghiên cứu. Trẻ em người Kinh 7 tuổi bao gồm 104 nam và 102 nũ.

\section{- Tiêu chuẩn lựa chọn:}

- Đối tượng là người dân tộc Kinh, có bố me, ông bà nội ngoại là người Kinh, không điêu trị chỉnh hình răng mặt trước và trong thời gian nghiên cứu, không có dị tật bẩm sinh, không có biến dạng xương hàm, không mắc bệnh ảnh hưởng đến tăng trưởng của cơ thể và vùng đầu - mặt, không có viêm nhiễm hoặc chấn thương nghiêm trọng vùng hàm mặt, trẻ và người thân của trẻ (cha mẹ hoặc người giám hộ) đồng ý tham gia nghiên cứu.

Đã mọc đầy đủ đủ bốn răng hàm lớn thứ nhất và chạm khớp hai hàm, khớp cắn theo phân loại Angle hai bên trái và phải giống nhau thể hiển cùng phân loại khớp cắn loại I, II và III Angle.

- Tiêu chuẩn loại trừ: Đối tượng không đủ các tiêu chuẩn lựa chọn.

- Thời gian và địa điểm nghiên cứu: Từ tháng 3/2017 đến tháng 12/2019 tại trường Tiểu học Liên Ninh, Thanh Trì, Hà Nội.

\subsection{Phương pháp nghiên cứu}

- Thiết kế nghiên cứu: Nghiên cứu dọc

- Vật liệu và phương tiện nghiên cứu: Máy ảnh Nikon 700D full frame, ống kính Nikkor AF-S 28-105 mm f/ 3,5-4,5D, giá đõ máy ảnh có thể trượt theo chiêu dọc, thước thủy bình có chia độ dài, thước thủy thăng bằng, giá kẹp thước thủy.

- Các điểm mốc giải phấu [6],[8]: Điểm cánh mũi (Alare: Al) điểm ngoài nhất của cánh mũi, điểm gò má (Zygion: Zy) điểm mô mềm ngoài cùng của xương gò má, điểm góc miệng (Cheilion: Ch) nơi gặp nhau của môi trên và môi dưới ở góc miệng, điểm góc hàm (Gonion: Go) điểm sau nhất và dưới nhất của góc hàm, điểm chân tóc (Trichion: $T r$ ) điểm giữa đường chân tóc của vùng trán, điểm trên gốc mũi (Glabella: GI) điểm nhồ nhất của vùng giữa trán, điểm dưới mũi (Subnasale: $\mathrm{Sn}$ ) điểm trên đường giữa chân mũi, nơi gặp nhau của mũi và môi trên, điểm dưới cằm (Mention: Me) điểm thấp nhất vùng cằm trên mặt phẳng dọc giữa.

- Các kích thước đo đạc [6],[9] (đơn vị: mm): Chiều rộng mũi (Al-Al), chiều rộng mặt (Zy-Zy), chiều rộng hàm dưới (Go-Go), chiêu rộng miệng (Ch-Ch), chiều cao tầng mặt trên ( $\mathrm{Tr}-\mathrm{Gl})$, chiều cao tâng mặt giữa (GI-Sn), chiều cao tâng mặt dưới (Sn-Me).

Xử lý số liệu: Số liệu sau khi thu thập được nhập liệu và̀ xử lý bằng phần mềm SPSS 23.0. Khi cần so sánh giá trị trung bình giữa hai giới hoặc hai nhóm sử dụng t-test hoặc MannWhitney test. Khi so sánh mức độ tăng trưởng và tỷ lệ tăng trưởng của hai nhóm tuổi 7-8 và 89 của từng loại khớp cắn thì sử dụng so sánh bắt cặp (paired - samples t test) hoặc Wilcoxon test; khi so sánh giá trị trung bình cùng giới giữa các loại khớp cắn thì sử dụng one way Anova test kết hợp Bonferoni test hoặc Kruskal Wallis test kết hợp Mann- Whitney test.

- Đạo đức trong nghiên cứu: Tiến hành nghiên cứu trên các đối tượng tự nguyện tham gia nghiên cứu, được Hội đồng đạo đức $Y$ sinh học của trường Đại học $Y$ Hà Nội cấp giấy chấp thuận số 47/HĐĐĐĐHYHN ngày 12/01/2017.

\section{KẾT QUẢ NGHIÊN CỨU}

Nghiên cứu 206 đối tượng trẻ em 7 tuổi dân tộc Kinh bao gồm 104 nam và 102 nữ bằng phương pháp đo trực tiếp cho kết quả như sau:

Bảng 1: Đặc điểm nhân trắc đầu mặt ở trẻ 7 tuổi dân tộc Kinh khi đo bằng kỹ thuật đo trên ảnh chuẩn hóa thẳng và nghiêng (mm)

\begin{tabular}{|c|c|c|c|c|c|c|}
\hline \multirow{2}{*}{\multicolumn{2}{|c|}{$\begin{array}{c}\text { Kích thước } \\
\text { đâu mặt }\end{array}$}} & \multicolumn{4}{|c|}{ Phân loại khớp căn theo Angle } & \multirow{2}{*}{$\mathbf{P}$} \\
\hline & & Loại I & Loại II & Loại III & Chung & \\
\hline \multirow{4}{*}{$\begin{array}{l}\text { Zy- } \\
\text { Zy }\end{array}$} & Nam & $122,56 \pm 5,06$ & $122,66 \pm 4,95$ & $122,00 \pm 4,90$ & $122,41 \pm 4,93$ & 0,7651 \\
\hline & Nữ & $119,27 \pm 4,22$ & $119,67 \pm 4,27$ & $119,59 \pm 3,96$ & $119,50 \pm 4,12$ & 0,5765 \\
\hline & $P_{1}$ & $0,0046 * *$ & $0,0022 * *$ & $0,0130 * *$ & $<0,001 * *$ & \\
\hline & Chung & $120,89 \pm 4,91$ & $121,21 \pm 4,84$ & $120,82 \pm 4,59$ & $120,97 \pm 4,76$ & 0,7122 \\
\hline $\begin{array}{l}\text { Al- } \\
\text { Al }\end{array}$ & Nam & $32,39 \pm 2,18$ & $33,06 \pm 2,34$ & $31,42 \pm 2,03$ & $32,31 \pm 2,27$ & $\begin{array}{c}\mathrm{P}_{\text {I-II }}>0,017 ; \mathbf{P}_{\text {I-IIII }} \mathbf{P}_{\text {III- }} \\
\text { III }<\mathbf{0 , 0 1 7}\end{array}$ \\
\hline
\end{tabular}


TẠP CHÍ Y HỌC VIẸT NAM TẬP 502 - THÁNG 5 - SÓ 2 - 2021

\begin{tabular}{|c|c|c|c|c|c|c|}
\hline & Nũ̃ & $29,89 \pm 1,93$ & $30,03 \pm 1,98$ & $28,53 \pm 1,83$ & $29,51 \pm 2,01$ & $\begin{array}{c}\mathrm{P}_{\mathrm{I}-\mathrm{II}}>0,017 ; \\
\text { III }<\mathbf{0 , 0 1 7}\end{array}$ \\
\hline & $\mathrm{P}_{1}$ & $<0,001 * *$ & $<0,001 * *$ & $<0,001 * *$ & $<0,001 * *$ & \\
\hline & Chung & $31,12 \pm 2,40$ & $31,59 \pm 2,64$ & $30,00 \pm 2,41$ & $30,92 \pm 2,56$ & $\begin{array}{c}\mathrm{P}_{\mathrm{IIII}}>0,017 ; \\
\text { III }<\mathbf{0 , 0 1 7}\end{array}$ \\
\hline \multirow{4}{*}{$\begin{array}{l}\text { Ch- } \\
\text { Ch }\end{array}$} & Nam & $39,03 \pm 2,94$ & $39,49 \pm 2,95$ & $38,76 \pm 2,57$ & $39,10 \pm 2,82$ & 0,5767 \\
\hline & Nữ & $37,05 \pm 2,38$ & $37,18 \pm 2,48$ & $36,75 \pm 2,24$ & $37,00 \pm 2,35$ & 0,6108 \\
\hline & $P_{1}$ & $0,0024^{*}$ & $<0,001 *$ & $0,0014 *$ & $<0,001 *$ & \\
\hline & Chung & $38,03 \pm 2,83$ & $38,37 \pm 2,95$ & $37,77 \pm 2,60$ & $38,06 \pm 2,80$ & 0,3498 \\
\hline \multirow{4}{*}{$\begin{array}{l}\text { Go- } \\
\text { Go }\end{array}$} & Nam & $91,69 \pm 4,49$ & $91,86 \pm 4,78$ & $90,70 \pm 4,86$ & $91,43 \pm 4,69$ & 0,4312 \\
\hline & Nữ & $89,03 \pm 4,54$ & $88,36 \pm 4,53$ & $88,53 \pm 4,28$ & $88,66 \pm 4,42$ & 0,5133 \\
\hline & $P_{1}$ & $0,0139 *$ & $0,0029 *$ & $0,0615 *$ & $<0,001 *$ & \\
\hline & Chung & $90,34 \pm 4,68$ & $90,16 \pm 4,95$ & $89,63 \pm 4,68$ & $90,06 \pm 4,76$ & 0,4408 \\
\hline \multirow{4}{*}{$\begin{array}{l}\text { Tr- } \\
\text { Gl }\end{array}$} & Nam & $53,53 \pm 3,73$ & $53,26 \pm 4,02$ & $52,91 \pm 3,84$ & $53,24 \pm 3,83$ & 0,6091 \\
\hline & Nữ & $51,14 \pm 3,38$ & $51,03 \pm 3,87$ & $50,84 \pm 3,54$ & $51,01 \pm 3,56$ & 0,8012 \\
\hline & $P_{1}$ & $0,0053 *$ & $0,0231 *$ & $0,0276 *$ & $<0,001^{*}$ & \\
\hline & Chung & $52,32 \pm 3,73$ & $52,18 \pm 4,07$ & $51,89 \pm 3,81$ & $52,14 \pm 3,86$ & 0,6577 \\
\hline \multirow{4}{*}{$\begin{array}{l}\mathrm{Gl}- \\
\mathrm{Sn}\end{array}$} & Nam & $56,22 \pm 3,78$ & $54,83 \pm 3,69$ & $58,09 \pm 3,98$ & $56,35 \pm 4,00$ & $\begin{array}{c}\mathrm{P}_{\text {I-II }}>0,017 ; \\
\text { III }<\mathbf{0 , 0 1 7}\end{array}$ \\
\hline & Nũ & $53,05 \pm 3,57$ & $\pm 3,36$ & $\pm 3,75$ & $=3,60$ & $\begin{array}{c}\text { PI-II }^{>0,017} ; \text { PI-III; } \text { PII- } \\
\text { III } 017\end{array}$ \\
\hline & $\mathrm{P}_{1}$ & $<0,001^{*}$ & $0,003^{*}$ & $<0,001^{*}$ & $<0,001^{*}$ & \\
\hline & Chung & $54,62 \pm 3,98$ & $53,54 \pm 3,75$ & $56,03 \pm 4,38$ & 54,71 & $\begin{array}{c}\mathrm{P}_{\text {I-II }}>0,017 ; \\
\text { III }<\mathbf{0}, \mathbf{0 1 7} \text { IIII }\end{array}$ \\
\hline \multirow{4}{*}{$\begin{array}{l}\mathrm{SN}- \\
\mathrm{Me}\end{array}$} & Nam & $58,03 \pm 4,27$ & $56,94 \pm 3,88$ & $59,97 \pm 4,43$ & $58,28 \pm 4,34$ & $\begin{array}{c}\mathrm{P}_{\mathrm{IIII}}>0,017 ; \mathbf{P}_{\mathrm{I}-\mathrm{III}} ; \mathbf{P}_{\text {II- }}<\mathbf{0 , 0 1 7} \\
\text { III }\end{array}$ \\
\hline & Nũ & $55,41 \pm 3,48$ & $53,91 \pm 3,36$ & $58,16 \pm 3,95$ & $55,78 \pm 3,96$ & $\begin{array}{c}\text { PI-II }^{>0,017 ;} ; \text { PIIIII; }_{\text {III }}<\mathbf{0 , 0 1 7} \\
\text { II- }\end{array}$ \\
\hline & $\mathrm{P}_{1}$ & $0,0019 * *$ & $<0,001 * *$ & $0,0141 * *$ & $<0,001 * *$ & \\
\hline & Chung & $56,70 \pm 4,08$ & $55,47 \pm 3,92$ & $59,08 \pm 4,27$ & $57,04 \pm 4,33$ & $\begin{array}{c}\mathrm{P}_{\mathrm{IIII}}>0,017 ; \\
\text { III }<\mathbf{0 , 0 1 7}\end{array}$ \\
\hline
\end{tabular}

P1: so sánh giá tri trung bình giữa hai giới khi so sánh theo giới tính trong cùng loai khớp tính (*: Sample t-test, **: Mann- Whitney test), $\mathrm{P}$ (One way ANOVA kết hợp Bonferoni test): So sánh giá trị trung bình giữa ba nhóm khớp cắn loại I, II và III Angle

Nhận xét: Bảng kết quả cho thấy có sự khác biệt có ý nghĩa thống kê giữa hai giới nam và nữ cắn Angle ở tất cả các biến số $(p<0,05)$. Khi so sánh giữa các loại khớp cắn ở cùng giới tính cho thấy có sự khác biệt có ý nghĩa thống kê ở kích thước chiều rộng mũi (Al-Al), chiều cao tầng mặt giữa (Gl-Sn), chiêu cao tâng mặt dưới (Sn-Me) $(\mathrm{p}<0,0017)$.

Bảng 2: Mức tăng trưởng và tỷ lệ gia tăng các kích thước vùng mặt ở các lửa tuổi từ 79 tuổi khớp cắn loại I Angle

\begin{tabular}{|c|c|c|c|c|c|c|}
\hline \multirow{2}{*}{\multicolumn{3}{|c|}{ Mức độ thay đổi }} & \multicolumn{3}{|c|}{ Tuối } & \multirow{2}{*}{$\mathbf{P}$} \\
\hline & & & $7-8$ & $8-9$ & $7-9$ & \\
\hline \multirow{8}{*}{ Zy-Zy } & \multirow{4}{*}{$\begin{array}{l}\text { Mức gia } \\
\text { tăng }(\mathrm{mm})\end{array}$} & Nam & $2,39 \pm 1,27$ & $2,61 \pm 1,32$ & $5,00 \pm 1,60$ & $0,5356^{* *}$ \\
\hline & & Nữ & $2,62 \pm 1,34$ & $2,89 \pm 1,33$ & $5,51 \pm 2,12$ & $0,4746 * *$ \\
\hline & & $P_{1}$ & $0,5221^{* *}$ & $0,2918^{* *}$ & $0,2248 * *$ & \\
\hline & & Chung & $2,51 \pm 1,30$ & $2,75 \pm 1,32$ & $5,26 \pm 1,89$ & $0,3267 * *$ \\
\hline & \multirow{4}{*}{$\begin{array}{c}\text { Tỷ lê gia } \\
\text { tăng (\%) }\end{array}$} & Nam & $1,96 \pm 1,06$ & $2,11 \pm 1,09$ & $4,11 \pm 1,40$ & $0,8875^{* *}$ \\
\hline & & Nũ̃ & $2,22 \pm 1,22$ & $2,38 \pm 1,11$ & $4,66 \pm 1,92$ & $0,8741^{* *}$ \\
\hline & & $P_{1}$ & $0,2225^{* *}$ & $0,0978^{* *}$ & $0,0774 * *$ & \\
\hline & & Chung & $2,09 \pm 1,15$ & $2,25 \pm 1,10$ & $4,39 \pm 1,70$ & $0,8885^{* *}$ \\
\hline \multirow{6}{*}{ Al-Al } & \multirow{4}{*}{$\begin{array}{l}\text { Mức gia } \\
\text { tăng }(\mathrm{mm})\end{array}$} & Nam & $2,22 \pm 0,93$ & $2,25 \pm 1,02$ & $4,47 \pm 1,03$ & $0,7007 * *$ \\
\hline & & Nữ & $2,43 \pm 0,90$ & $2,51 \pm 0,99$ & $4,95 \pm 1,45$ & $0,6857^{* *}$ \\
\hline & & $P_{1}$ & $0,3292 *$ & $0,1421^{* *}$ & $0,1129^{*}$ & \\
\hline & & Chung & $2,33 \pm 0,91$ & $2,38 \pm 1,01$ & $4,71 \pm 1,27$ & $0,9573 * *$ \\
\hline & \multirow{2}{*}{$\begin{array}{c}\text { Tỷ lệ gia } \\
\text { tăng }(\%)\end{array}$} & Nam & $6,90 \pm 2,98$ & $6,57 \pm 3,11$ & $13,88 \pm 3,39$ & $0,2386 * *$ \\
\hline & & Nữ & $8,22 \pm 3,19$ & $7,81 \pm 3,09$ & $16,68 \pm 5,17$ & $0,4195^{* *}$ \\
\hline
\end{tabular}




\begin{tabular}{|c|c|c|c|c|c|c|}
\hline & & $P_{1}$ & $0,0032 *$ & $0,0077 * *$ & $<0,001^{*}$ & \\
\hline & & Chung & $7,57 \pm 3,14$ & $7,20 \pm 3,14$ & $15,30 \pm 4,58$ & $0,1327^{* *}$ \\
\hline \multirow{8}{*}{ Ch-Ch } & \multirow{4}{*}{$\begin{array}{l}\text { Mức gia } \\
\text { tăng }(\mathrm{mm})\end{array}$} & Nam & $2,28 \pm 0,88$ & $2,31 \pm 0,86$ & $4,58 \pm 1,05$ & $0,9048^{*}$ \\
\hline & & Nữ & $2,41 \pm 0,86$ & $2,35 \pm 0,89$ & $4,76 \pm 1,32$ & $0,7772 *$ \\
\hline & & $\mathrm{P}_{1}$ & 0,5344* & 0,8233* & 0,5376* & \\
\hline & & Chung & $2,34 \pm 0,87$ & $2,33 \pm 0,87$ & $4,67 \pm 1,19$ & $0,9265^{*}$ \\
\hline & \multirow{4}{*}{$\begin{array}{l}\text { Tỷ lẹ gia } \\
\text { tăng }(\%)\end{array}$} & Nam & $5,83 \pm 2,20$ & $5,67 \pm 2,25$ & $11,82 \pm 2,85$ & $0,7768^{*}$ \\
\hline & & Nữ & $6,54 \pm 2,45$ & $5,97 \pm 2,24$ & $12,91 \pm 3,79$ & $0,2704 *$ \\
\hline & & $P_{1}$ & $0,1996 *$ & $0,5594 *$ & $0,2507 * *$ & \\
\hline & & Chung & $6,19 \pm 2,34$ & $5,82 \pm 2,24$ & $12,37 \pm 3,38$ & $0,3409 *$ \\
\hline \multirow{8}{*}{ Go-Go } & \multirow{4}{*}{$\begin{array}{l}\text { Mức gia } \\
\text { tăng }(\mathrm{mm})\end{array}$} & Nam & $2,33 \pm 0,83$ & $2,39 \pm 0,84$ & $4,72 \pm 1,19$ & $0,7773^{*}$ \\
\hline & & Nữ & $2,54 \pm 0,87$ & $2,57 \pm 0,93$ & $5,11 \pm 1,26$ & $0,8985^{*}$ \\
\hline & & $P_{1}$ & $0,3008^{*}$ & $0,3915^{*}$ & $0,1832^{*}$ & \\
\hline & & Chung & $2,44 \pm 0,85$ & $2,48 \pm 0,88$ & $4,92 \pm 1,23$ & $0,7740^{*}$ \\
\hline & \multirow{4}{*}{$\begin{array}{l}\text { Tỷ lệ gia } \\
\text { tăng }(\%)\end{array}$} & Nam & $2,55 \pm 0,92$ & $2,54 \pm 0,88$ & $5,16 \pm 1,29$ & $0.9685^{*}$ \\
\hline & & Nữ & $2,86 \pm 0,98$ & $2,81 \pm 1,03$ & $5,76 \pm 1,46$ & $0.8349 *$ \\
\hline & & $P_{1}$ & $0,1659 *$ & $0,2318^{*}$ & $0,0683^{*}$ & \\
\hline & & Chung & $2.71 \pm 0.96$ & $2,68 \pm 0,96$ & $5,46 \pm 1,40$ & $0,8537 *$ \\
\hline \multirow{8}{*}{ Tr-Gl } & \multirow{4}{*}{$\begin{array}{l}\text { Mức gia } \\
\text { tăng }(\mathrm{mm})\end{array}$} & Nam & $1,28 \pm 0,45$ & $1,31 \pm 0,52$ & $2,58 \pm 0,65$ & $0,9442^{* *}$ \\
\hline & & Nữ & $1,30 \pm 0,52$ & $1,32 \pm 0,53$ & $2,62 \pm 0,64$ & $0,8258^{* *}$ \\
\hline & & $P_{1}$ & $0,9899 * *$ & $0,8611^{* *}$ & $0,7679 * *$ & \\
\hline & & Chung & $1,29 \pm 0,49$ & $1,32 \pm 0,52$ & $2,60 \pm 0,64$ & $0,8286 * *$ \\
\hline & \multirow{4}{*}{$\begin{array}{l}\text { Tỷ lệ gia } \\
\text { tăng }(\%)\end{array}$} & Nam & $2,40 \pm 0,89$ & $2,40 \pm 1,01$ & $4,86 \pm 1,30$ & $0,0552^{* *}$ \\
\hline & & Nữ & $2,56 \pm 1,07$ & $2,54 \pm 1,05$ & $5,17 \pm 1,37$ & $0,1583^{* *}$ \\
\hline & & $P_{1}$ & $0,1127^{* *}$ & $0,0846^{* *}$ & $0,1479 * *$ & \\
\hline & & Chung & $2,48 \pm 0,98$ & $2,47 \pm 1,03$ & $5,02 \pm 1,33$ & $0,0623 * *$ \\
\hline \multirow{8}{*}{ Gl-Sn } & \multirow{4}{*}{$\begin{array}{l}\text { Mức gia } \\
\text { tăng }(\mathrm{mm})\end{array}$} & $\mathrm{Nam}$ & $1,89 \pm 0,82$ & $2,00 \pm 0,83$ & $3,89 \pm 1,30$ & $0,4607^{* *}$ \\
\hline & & Nũ̃ & $2,03 \pm 0,83$ & $2,11 \pm 0,81$ & $4,14 \pm 0,98$ & $0,8645^{* *}$ \\
\hline & & $P_{1}$ & $0,4558^{* *}$ & $0,5744^{*}$ & $0,3634^{*}$ & \\
\hline & & Chung & $1,96 \pm 0,82$ & $2,05 \pm 0,81$ & $4,01 \pm 1,15$ & $0,5623^{* *}$ \\
\hline & \multirow{4}{*}{$\begin{array}{l}\text { Tỷ lệ gia } \\
\text { tăng }(\%)\end{array}$} & Nam & $3,43 \pm 1,61$ & $3,49 \pm 1,54$ & $7,05 \pm 2,67$ & $0,5194 * *$ \\
\hline & & Nữ & $3,89 \pm 1,77$ & $3,86 \pm 1,54$ & $7,89 \pm 2,19$ & $0,3774 * *$ \\
\hline & & $P_{1}$ & $0,0807^{* *}$ & $0,0839 * *$ & $0,1454 *$ & \\
\hline & & Chung & $3,66 \pm 1,70$ & $3,68 \pm 1,54$ & $7,48 \pm 2,46$ & $0,2776^{* *}$ \\
\hline \multirow{8}{*}{ Sn-Me } & \multirow{4}{*}{$\begin{array}{l}\text { Mức gia } \\
\text { tăng }(\mathrm{mm})\end{array}$} & Nam & $2,31 \pm 0,75$ & $2,42 \pm 0,77$ & $4,72 \pm 0,97$ & $0,5710^{*}$ \\
\hline & & Nữ & $2,51 \pm 0,77$ & $2,65 \pm 0,79$ & $5,16 \pm 1,12$ & $0,4533^{*}$ \\
\hline & & $P_{1}$ & $0,2457 *$ & $0,2080^{*}$ & $0,0777^{*}$ & \\
\hline & & Chung & $2,41 \pm 0,76$ & $2,53 \pm 0,78$ & $4,95 \pm 1,07$ & $0,3490 *$ \\
\hline & \multirow{4}{*}{$\begin{array}{l}\text { Tỷ lệ gia } \\
\text { tăng }(\%)\end{array}$} & Nam & $4,01 \pm 1,37$ & $4,02 \pm 1,28$ & $8,19 \pm 1,82$ & $0,9740 *$ \\
\hline & & Nữ & $4,55 \pm 1,40$ & $4,62 \pm 1,48$ & $9,38 \pm 2,20$ & $0,8415^{*}$ \\
\hline & & $P_{1}$ & $0,0997 *$ & $0,0701^{*}$ & $0,0144 *$ & \\
\hline & & Chung & $4,29 \pm 1,40$ & $4,32 \pm 1,41$ & $8,80 \pm 2,10$ & $0,8678^{*}$ \\
\hline \multicolumn{4}{|c|}{$\begin{array}{l}\text { P1: so sánh giá trị trung bình giữa hai giới tính } \\
\text { (*: Sample t-test, **: Mann- Whitney test) , P: So } \\
\text { sánh mức gia tăng và tỳ lệ gia tăng tứ 7-8 tuổi và } \\
\text { từ 8-9 tuổi (*: paired t-test, **: Wilcoxon test) } \\
\text { Nhấn xét: Mức tăng trưởng và tỷ lệ gia tăng } \\
\text { các kích thước vùng mặt không có sự khác biệt }\end{array}$} & \multicolumn{3}{|c|}{$\begin{array}{l}\text { khi so sánh giữa hai giới tính nam và nữ, trừ tỷ } \\
\text { lệ gia tăng chiêuu cao tầng mặt dưới từ } 7-9 \text { tuổi ỡ } \\
\text { nữ lớn hơn ở nam, tỳ lệ gia tăng chiều rộng mũi } \\
\text { (Al-Al) ở nữ từ } 8-9 \text { tuổi và } 7-9 \text { tuổi lớn hơn ở } \\
\text { nam giới }(p<0,05) \text {. }\end{array}$} \\
\hline \multicolumn{7}{|c|}{$\begin{array}{l}\text { Bảng 3: Mức tăng trưởng và tỷ lệ gia tăng các kích thước vùng mặt ở các lửa tuổi từ 7- } \\
\text { tuối khớp cắn loại II Angle }\end{array}$} \\
\hline \multirow{2}{*}{\multicolumn{3}{|c|}{ Mức độ thay đổi }} & \multicolumn{3}{|c|}{ Tuối } & P \\
\hline & & & $7-8$ & $8-9$ & $7-9$ & $P$ \\
\hline \multirow{3}{*}{ Zy-Zy } & & Nam & $2,37 \pm 1,24$ & $2,60 \pm 1,26$ & $4,97 \pm 1,98$ & $0,4575^{* *}$ \\
\hline & tăng $(\mathrm{mm})$ & Nữ & $2,61 \pm 1,27$ & $2,91 \pm 1,28$ & $5,52 \pm 1,99$ & $0,2045^{* *}$ \\
\hline & & $P_{1}$ & $0,3689 * *$ & $0,2920^{* *}$ & $0,1297 * *$ & \\
\hline
\end{tabular}


TẠP CHÍ Y HỌC VIẸT NAM TẬP 502 - THÁNG 5 - SÓ 2 - 2021

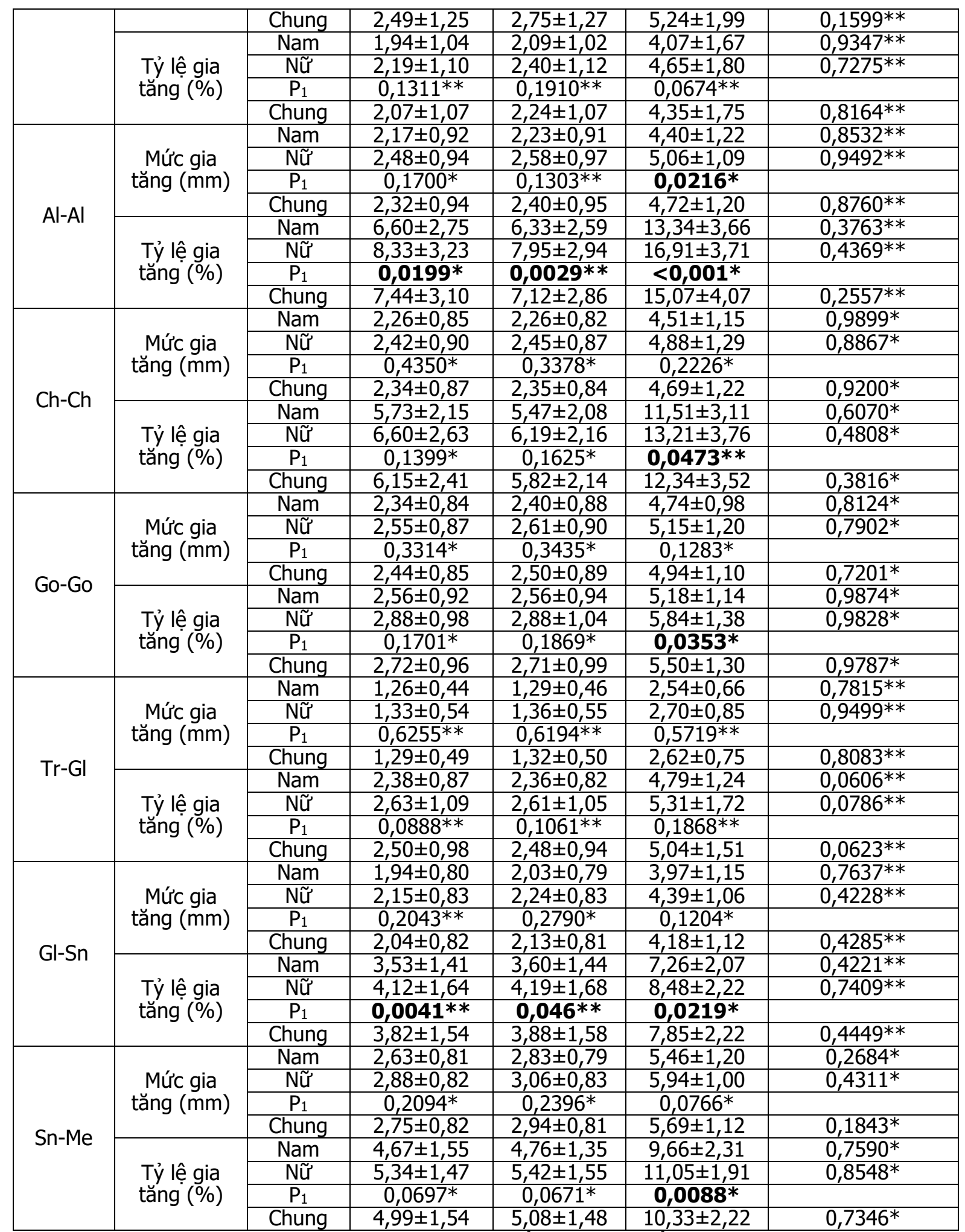

P1: so sánh giá trị trung bình giữa hai giới tuối và từ 8-9 tuối (*: paired t-test, **: Wilcoxon tính (*: Sample t-test, **: Mann- Whitney test), test)

P: So sánh mức gia tăng và tỷ lệ gia tăng từ 7-8 Nhận xét: Mức tăng trưởng và tỷ lệ gia tăng 
các kích thước vùng mặt ở các lửa tuổi từ 7-9 tuổi khớp cắn loại II Angle khi so sánh giữa hai giới ở hầu hết các kích thước, tuy nhiên, tỷ lệ gia tăng ở các kích thước chiêu rộng mũi (Al-Al), chiêuu rộng hàm dưới (Go-Go), chiều cao tầng mặt giữa (Gl-Sn), chiều cao tầng mặt dưới (Sn$\mathrm{Me}$ ) từ 7-9 tuổi ở nữ giới lớn hơn nam giới có ý nghĩa thống kê $(p<0,05)$.

Bảng 4: Mức tăng trưởng và tỷ lệ gia tăng các kích thước vùng mặt ở các lửa tuổi từ 79 tuổi khớp cắn loại III Angle

\begin{tabular}{|c|c|c|c|c|c|c|}
\hline \multirow{2}{*}{\multicolumn{3}{|c|}{ Mức độ thay đổi }} & \multicolumn{3}{|c|}{ Tuối } & \multirow{3}{*}{$\begin{array}{c}\mathbf{P} \\
0,7104^{* *}\end{array}$} \\
\hline & & & 7-8 & 8-9 & 7-9 & \\
\hline \multirow{8}{*}{$Z y-Z y$} & \multirow{4}{*}{$\begin{array}{l}\text { Mức gia } \\
\text { tăng (mm) }\end{array}$} & Nam & $2,42 \pm 1,25$ & $2,61 \pm 1,25$ & $5,03 \pm 1,59$ & \\
\hline & & Nữ & $2,69 \pm 1,28$ & $2,97 \pm 1,31$ & $5,66 \pm 1,70$ & $0,6090 * *$ \\
\hline & & $P_{1}$ & $0,3579 * *$ & $0,2365^{* *}$ & $0,0740 * *$ & \\
\hline & & Chung & $2,55 \pm 1,26$ & $2,78 \pm 1,28$ & $5,34 \pm 1,66$ & $0,5273^{* *}$ \\
\hline & \multirow{4}{*}{$\begin{array}{l}\text { Tỷ lệ gia } \\
\text { tăng (\%) }\end{array}$} & $\mathrm{Nam}$ & $2,01 \pm 1,08$ & $2,10 \pm 1,00$ & $4,14 \pm 1,37$ & $0,7681^{* *}$ \\
\hline & & Nữ & $2,27 \pm 1,12$ & $2,43 \pm 1,07$ & $4,75 \pm 1,49$ & $0,8811^{* *}$ \\
\hline & & $P_{1}$ & $0,1245^{* *}$ & $0,0941^{* *}$ & $0,0194 * *$ & \\
\hline & & Chung & $2,13 \pm 1,10$ & $2,26 \pm 1,04$ & $4,44 \pm 1,45$ & $0,7662^{* *}$ \\
\hline \multirow{8}{*}{ Al-Al } & \multirow{4}{*}{$\begin{array}{l}\text { Mức gia } \\
\text { tăng (mm) }\end{array}$} & Nam & $2,24 \pm 0,94$ & $2,27 \pm 0,91$ & $4,52 \pm 1,12$ & $0,8162^{* *}$ \\
\hline & & Nữ & $2,44 \pm 0,91$ & $2,53 \pm 1,02$ & $4,97 \pm 1,40$ & $0,8161^{* *}$ \\
\hline & & $P_{1}$ & $0,3987^{*}$ & $0,3148^{* *}$ & $0,1541^{*}$ & \\
\hline & & Chung & $2,34 \pm 0,92$ & $2,40 \pm 0,97$ & $4,74 \pm 1,28$ & $0,7398^{* *}$ \\
\hline & \multirow{4}{*}{$\begin{array}{l}\text { Tỷ lệ gia } \\
\text { tăng (\%) }\end{array}$} & Nam & $7,11 \pm 2,85$ & $6,84 \pm 2,89$ & $14,41 \pm 3,70$ & $0,4369 * *$ \\
\hline & & Nữ & $8,52 \pm 3,17$ & $8,23 \pm 3,36$ & $17,45 \pm 5,00$ & $0,4001^{* *}$ \\
\hline & & $P_{1}$ & $0,0235 *$ & $0,0327 * *$ & $0,0070 *$ & \\
\hline & & Chung & $7,80 \pm 3,07$ & $7,52 \pm 3,19$ & $15,91 \pm 4,61$ & $0,2853 * *$ \\
\hline \multirow{8}{*}{ Ch-Ch } & \multirow{4}{*}{$\begin{array}{l}\text { Mức gia } \\
\text { tăng (mm) }\end{array}$} & Nam & $2,27 \pm 0,88$ & $2,30 \pm 0,88$ & $4,58 \pm 1,39$ & $0,8724 *$ \\
\hline & & Nữ & $2,44 \pm 0,95$ & $2,47 \pm 0,92$ & $4,91 \pm 1,28$ & $0,8971^{*}$ \\
\hline & & $P_{1}$ & 0,4693* & $0,4603^{*}$ & $0,3232 *$ & \\
\hline & & Chung & $2,35 \pm 0,91$ & $2,38 \pm 0,90$ & $4,74 \pm 1,34$ & $0,8384 *$ \\
\hline & \multirow{4}{*}{$\begin{array}{l}\text { Tỷ lệ gia } \\
\text { tăng }(\%)\end{array}$} & Nam & $5,88 \pm 2,35$ & $5,67 \pm 2,27$ & $11,90 \pm 3,98$ & $0,6688^{*}$ \\
\hline & & Nữ & $6,68 \pm 2,61$ & $6,35 \pm 2,48$ & $13,44 \pm 3,67$ & $0,6214 *$ \\
\hline & & $P_{1}$ & 0,0209* & $0,0252 *$ & $0,0339 * *$ & \\
\hline & & Chung & $6,27 \pm 2,50$ & $6,01 \pm 2,38$ & $12,66 \pm 3,88$ & $0,5097^{*}$ \\
\hline \multirow{8}{*}{ Go-Go } & \multirow{4}{*}{$\begin{array}{l}\text { Mức gia } \\
\text { tăng (mm) }\end{array}$} & Nam & $2,30 \pm 0,77$ & $2,36 \pm 0,86$ & $4,67 \pm 1,27$ & 0,7373* \\
\hline & & Nũ & $2,53 \pm 0,88$ & $2,59 \pm 0,91$ & $5,13 \pm 1,10$ & $0,8040 *$ \\
\hline & & $P_{1}$ & 0,2694* & $0,2987 *$ & $0,1248^{*}$ & \\
\hline & & Chung & $2,42 \pm 0,83$ & $2,48 \pm 0,89$ & $4,89 \pm 1,20$ & $0,6864 *$ \\
\hline & \multirow{4}{*}{$\begin{array}{l}\text { Tỷ lệ gia } \\
\text { tăng }(\%)\end{array}$} & Nam & $2,55 \pm 0,87$ & $2,55 \pm 0,94$ & $5,17 \pm 1,45$ & 0,9915* \\
\hline & & Nữ & $2,87 \pm 1,00$ & $2,85 \pm 1,00$ & $5,80 \pm 1,26$ & $0,9350 *$ \\
\hline & & $\mathrm{P}_{1}$ & $0,1760^{*}$ & $0,2203^{*}$ & $0,0673^{*}$ & \\
\hline & & Chung & $2,71 \pm 0,94$ & $2,70 \pm 0,97$ & $5,48 \pm 1,38$ & 0,9417* \\
\hline \multirow{8}{*}{ Tr-Gl } & \multirow{4}{*}{$\begin{array}{l}\text { Mức gia } \\
\text { tăng (mm) }\end{array}$} & Nam & $1,30 \pm 0,47$ & $1,33 \pm 0,48$ & $2,64 \pm 0,70$ & $0,7815^{* *}$ \\
\hline & & Nũ̃ & $1,34 \pm 0,48$ & $1,38 \pm 0,55$ & $2,72 \pm 0,68$ & $0,9351^{* *}$ \\
\hline & & $\mathrm{P}_{1}$ & $0,7277^{* *}$ & $0,8607^{* *}$ & $0,5890 * *$ & \\
\hline & & Chung & $1,32 \pm 0,47$ & $1,35 \pm 0,51$ & $2,68 \pm 0,69$ & $0,8132 * *$ \\
\hline & \multirow{4}{*}{$\begin{array}{l}\text { Tỷ lệ gia } \\
\text { tăng (\%) }\end{array}$} & Nam & $2,48 \pm 0,91$ & $2,48 \pm 0,92$ & $5,01 \pm 1,39$ & $0,0643^{* *}$ \\
\hline & & Nữ & $2,64 \pm 0,91$ & $2,63 \pm 0,99$ & $5,33 \pm 1,21$ & $0,1395^{* *}$ \\
\hline & & $\mathrm{P}_{1}$ & $0,0885^{* *}$ & $0,1237^{* *}$ & $0,148^{* *}$ & \\
\hline & & Chung & $2,56 \pm 0,90$ & $2,55 \pm 0,95$ & $5,17 \pm 1,30$ & $0,0801^{* *}$ \\
\hline \multirow{7}{*}{$\mathrm{Gl}-\mathrm{Sn}$} & \multirow{4}{*}{$\begin{array}{l}\text { Mức gia } \\
\text { tăng (mm) }\end{array}$} & Nam & $2,03 \pm 0,81$ & $2,06 \pm 0,83$ & $4,09 \pm 1,13$ & $0,6027^{* *}$ \\
\hline & & Nũ̃ & $2,22 \pm 0,83$ & $2,28 \pm 0,81$ & $4,50 \pm 0,92$ & $0,6094 * *$ \\
\hline & & $\mathrm{P}_{1}$ & $0,2766^{* *}$ & $0,2822^{*}$ & $0,1141^{*}$ & \\
\hline & & Chung & $2,12 \pm 0,82$ & $2,17 \pm 0,82$ & $4,29 \pm 1,04$ & $0,466^{* *}$ \\
\hline & \multirow{3}{*}{$\begin{array}{l}\text { Tỷ lệ gia } \\
\text { tăng (\%) }\end{array}$} & Nam & $3,55 \pm 1,51$ & $3,44 \pm 1,43$ & $7,11 \pm 2,13$ & $0,4802^{* *}$ \\
\hline & & Nữ & $4,16 \pm 1,61$ & $4,10 \pm 1,52$ & $8,43 \pm 1,93$ & $0,6671 * *$ \\
\hline & & $P_{1}$ & $0,0045 * *$ & $0,0088 * *$ & $0,0113 *$ & \\
\hline
\end{tabular}




\begin{tabular}{|c|c|c|c|c|c|c|}
\hline & & Chung & $3,85 \pm 1,58$ & $3,77 \pm 1,50$ & $7,76 \pm 2,12$ & $0,4543 * *$ \\
\hline \multirow{8}{*}{ Sn-Me } & \multirow{4}{*}{$\begin{array}{l}\text { Mức gia } \\
\text { tăng (mm) }\end{array}$} & Nam & $2,97 \pm 0,77$ & $3,09 \pm 0,80$ & $6,06 \pm 1,22$ & $0,4880^{*}$ \\
\hline & & Nữ & $3,06 \pm 0,80$ & $3,25 \pm 0,84$ & $6,31 \pm 1,12$ & $0,3840 *$ \\
\hline & & $P_{1}$ & $0,6355^{*}$ & $0,4391 *$ & $0,3902^{*}$ & \\
\hline & & Chung & $3,02 \pm 0,78$ & $3,17 \pm 0,82$ & $6,18 \pm 1,17$ & 0,2607* \\
\hline & \multirow{4}{*}{$\begin{array}{l}\text { Tỷ lệ gia } \\
\text { tăng (\%) }\end{array}$} & Nam & $5,02 \pm 1,45$ & $4,94 \pm 1,40$ & $10,22 \pm 2,46$ & $0,8019 *$ \\
\hline & & Nữ & $5,29 \pm 1,44$ & $5,37 \pm 1,51$ & $10,94 \pm 2,22$ & $0,8285^{*}$ \\
\hline & & $P_{1}$ & $0,4544^{*}$ & $0,2471^{*}$ & $0,2189^{*}$ & \\
\hline & & Chung & $5,15 \pm 1,44$ & $5,15 \pm 1,46$ & $10,57 \pm 2,35$ & $0,9916^{*}$ \\
\hline
\end{tabular}

P1: so sánh giá trị trung bình giữa hai giới tính (*: Sample t-test, **: Mann- Whitney test) , P: So sánh mức gia tăng và tỷ lệ gia tăng từ 7-8 tuổi và từ 8-9 tuổi (*: paired t-test, **: Wilcoxon test)

Nhận xét: Mức tăng trưởng và tỷ lệ gia tăng các kích thước vùng mặt ở các lửa tuổi từ 7-9 tuổi khớp cắn loai III Angle đa số không có sự khác biệt giữa hai giới nam và nữ, không có sự khác biệt giữa hai khoảng tuổi 7-8 tuổi và 8-9 tuổi, trừ tỷ lệ gia tăng các kích thước chiều rộng mặt (Zy-Zy), chiều rộng mũi (Al-Al), chiều rộng miệng (Ch-Ch), chiêu cao tầng mặt giữa (Gl-Sn) từ 7-9 tuổi ở nứ lớn hơn ở nam giới $(\mathrm{p}<0,05)$.

\section{BÀN LUẬN}

Theo kết quả nghiên cứu, các kích thước vùng mặt khi đo trên ảnh chuẩn hóa kỹ thuật số thẳng và nghiêng, chiều rộng mặt $(Z y-Z y)$, chiều rộng mũi (Al-Al), chiều rộng miệng (Ch-Ch), chiều rộng hàm dưới (Go-Go), chiều cao tầng mặt trên (Tr-Gl), chiều cao tầng mặt giữa (Gl$\mathrm{Sn})$, chiều cao tầng mặt dưới $(\mathrm{SN}-\mathrm{Me})$ ở nam giới lớn hơn ở nữ giới có ý nghĩa thống kê, kết quả này phù hợp với nghiên cứu của Lê Đức Lánh (2007) [4] khi đo ở trẻ 12-15 tuổi, phù hợp với nghiên cứu của Võ Trương Như Ngọc (2010) [5], Trần Tuấn Anh (2016) [6] khi nghiên cứu ở người trưởng thành 18-25 tuổi. Khi so sánh giữa ba nhóm khớp cắn I, II và III theo phân loại của Angle, kích thước chiều rộng mũi (Al-Al) ở trẻ 7 tuổi nhóm loại III angle nhỏ hơn so với loại I và II Angle; chiều cao tầng mă̆t giữa (GI-Sn), chiều cao tâng mặt dưới (Sn-Me) ở trẻ 7 tuổi loại III angle lớn hớn so với loại I và II angle, điều này có thể do các đối tượng nghiên cứu thuộc loại III angle có góc tăng trưởng góc mở (loại III góc cao) nên có xu hướng tăng trưởng ra trước và xuống dưới của xương hàm dưới diễn ra mạnh mẽ hơn so với loại I và loại II angle (bảng 1).

Sự tăng trưởng kích thước vùng mặt trẻ từ 79 tuổi ở loại I angle, mức gia tăng và tỷ lệ tăng trưởng các kích thước không có sự khác biệt có ý nghĩa thống kê khi so sánh giữa hai giới nam và nữ ở khoảng 7-8 tuổi, 8-9 tuổi và 7-9 tuổi, mặc dù kích thước các chỉ số ở nữ có xu hướng lớn hơn ở nam, tuy nhiên, tỷ lệ gia tăng kích thước chiều rộng mũi (Al-Al), chiều cao tầng mặt dưới
(Sn-Me) từ 7-9 tuổi ở nữ lớn hơn ở nam, không có sự khác biệt có ý nghĩa thống kê về mức gia tăng và tỷ lệ tăng trưởng từ 7-8 tuổi và $8-9$ tuổi ở cả hai giới, khoảng tăng trưởng 8-9 tuổi có xu hướng lớn hơn khoảng tăng trưởng 7-8 tuổi (bảng 2). Sự tăng trưởng kích thước vùng mặt trẻ từ 7-9 tuổi ở loai II angle tương tứ như loai I angle, tuy nhiên, tỷ lệ gia tăng ở nữ lớn hơn ở nam giới các kích thước chiều rộng mũi, chiều rộng miệng, chiều rộng hàm dưới, chiều cao tầng mặt giữa, chiều cao tầng mặt dưới có ý nghĩa thống kê (bảng 3). Sự tăng trưởng kích thước vùng mặt trẻ từ 7-9 tuổi ở loại III angle tương tự như loại I và II angle, các kích thước chiều rộng mặt, chiều rộng mũi, chiều rộng miệng, chiều cao tầng mặt giữa ở nữ có tỷ lệ gia tăng lớn hơn ở nam giới có ý nghĩa thống kê (bảng 4). Đặc điểm tăng trưởng kích thước vùng mặt phù hợp với nghiên cứu của Lê Đức Lánh (2007) [4] cho thấy có sự tăng trưởng đều đặn từ 7-9 tuổi, tương tự tăng trưởng từ 12-15 tuổi, tuy nhiên, mức gia tăng và tỷ lệ gia tăng từ 7-9 tuổi ở nữ có xu hướng lớn hơn ở nam giới, và khoảng 8-9 tuổi có xu hướng lớn hơn 7-8 tuổi. Các kích thước vùng đầu mặt khi so sánh các chỉ số theo chiều ngang trong nghiên cứu của Trương Hoàng Lệ Thủy, Nguyễn Thị Kim Anh (2012) [6] cho thấy tương đồng giữa hai nghiên cứu, mức độ gia tăng và tỷ lệ gia tăng ở khoảng 8-9 tuổi có xu hướng lớn hớn khoảng 7-8 tuổi, điều này có thể do khoảng tuổi 8-9 tuổi gần đỉnh tăng trưởng hơn khoảng tuổi 7-8 tuổi.

\section{KẾT LUẬN}

Qua nghiên cứu dọc thực hiện bằng phương pháp đo trên ảnh chuẩn hóa thẳng và nghiêng nhân trắc đầu - mặt trên 206 trẻ em 7 -9 tuổi người Kinh (104 nam, 102 nữ), rút ra kết luận sau: Các kích thước chiều rộng mặt, chiều rộng mũi, chiều rộng miệng, chiều rộng hàm dưới, chiều cao tầng mặt trên, chiều cao tầng mặt giữa, chiều cao tầng mặt dưới ở nam lớn hởn nữ, khác biệt có ý nghĩa thống kê ở trẻ 7 tuổi. 
Sự tăng trưởng các kích thước vùng đầu mặt diễn ra liên tục và đều đặn từ 7-9 tuổi, ở nữ có xu hướng tăng trưởng sớm hơn, nhanh hơn ở nam giới khi so sánh giữa nam và nữ ở cùng khoảng tuổi 7-8 tuổi và 8-9 tuổi, ở mỗi loại khớp cắn theo phân loại Angle.

Lời cảm ơn. Trân trọng cảm ơn những đối tượng tự nguyện tham gia nghiên cứu, cảm ơn sự giúp đõ của Ban giám hiệu trường Tiểu học Liên Ninh, Thanh Trì, Hà Nội. Xin chân thành cảm ơn Viện Răng Hàm Mặt - Đại học Y Hà Nội đã tạo điều kiện cho chúng tôi hoàn thành nghiên cứu này.

\section{TÀI LIẸU THAM KHẢO}

1. Farhad.B Naini (2011). Facial aesthetics: Concepts and Clinnical diagnosis, John Wiley \& Sons Ltd, Chichester, chapter 2, 18-44.

2. J.Leon Williams (1920). New classification of human tooth forns with special reference to a new system of artificial teeth, The dentist's supply .Co, NewYork, 49-124.

3. Ibrahimagic.L, Jerolimov.V, Celebie.A et al (2001). Ralationship between the face and the tooth form, coll. Antropol, 25(2), 619-626.

4. Lê Đức Lánh (2007). Sự phát triển hình thái đầu mặt của trẻ em Viêt Nam từ 12-15 tuổi. Y học Thành phố Hồ Chí Minh, 11(2), 68-78.

5. Võ Trương Như Ngọc (2010). Nghiên cứu đăc điểm kết câu sọ mặt và đánh giá khuôn mặt hài hòa ở một nhóm người Viêtt tuôii từ 18-25, Luần án tiến sỹ y hơc, Viên Đào Tạo Răng Hàm Mặt- Đại học Y Ha Nội, 4-154.

6. Trương Hoàng Lê Thủy, Nguyễn Thị Kim Anh (2012). Sự thay đổi hình thái vùng mặt ở trẻ em Viêt Nam từ 6 đến 12 tuổi. $Y$ học Thành phố Hồ Chí Minh, 16(2), 69-77.

7. Trânn Tuấn Anh (2016). Nghiên cứu một số đặc điểm hình thái, chỉ số đâu măt ở môt nhóm người Việt độ tuổi từ 18-25 có khớp cắn bình thường và khiôn mặt hài hòa, Luận văn tiến sỹ sinh học, Trường Đại học Y Hà Nội, 63-108.

8. Claman $L$ et al (1990). Standardized portrait photography for dental patients, The American journal of Orthodontics and Dentofacial Orthopedics, 98(3), 197-205.

9. Bishara S. E et al (1995). Changes in facial dimensions assessed from lateral and frontal photographs, methodology, The American journal of Orthodontics and Dentofacial Orthopedics, 108(4), 389-393.

\section{KHẢO SÁT CƠ CẤU DANH MỤC SẢN PHẨM SẢN XUẤT KINH DOANH CỦA CÔNG TY CỔ PHẦN DƯỢC KHOA, GIAI ĐOAN 2015-2019}

\section{Nguyễn Thị Song Hà*}

\section{TÓM TẮT}

Mục tiêu nghiên cứu: Khảo sát cơ câu danh mục sản phẩm kinh doanh của Công ty cổ phân Dược Khoa giai đoạn 2015-2019. Đối tượng nghiên cứu: Danh mục sản phẩm và một số kết quả sản xuất kinh doanh của Công ty cổ phần Dược Khoa từ năm 2015 đến hết năm 2019. Phương pháp nghiên cứu: Nghiên cứu mô tả cắt ngang. b Cơ cấu danh mục sản phẩm của Công ty giai đoạn 2015-2019 gồm 4 nhóm sản phẩm: Thuốc, TPBVSK (Thực phẩm bảo vệ sức khỏe), Mỹ phẩm và TTBYT (Trang thiết bị Y tế). Nhóm TPBVSK luôn có số sản phẩm kinh doanh lớn nhất với trên $50 \%$ số sản phẩm kinh doanh hàng năm. Nhóm Thuốc có số sản phẩm kinh doanh đứng thứ 2 với khoảng $20 \%$ sản phẩm hàng năm, nhưng là nhóm chiếm tỷ trọng doanh thu chính với trên $40 \%$ tổng doanh thu sản xuất. Nhóm Mỹ phẩm và TTBYT có sự phát triển mạnh về số lượng sản phẩm kinh doanh và doanh thu qua các năm giai đoạn 2015-2019. Kết luận: Nhóm sản phẩm Thuốc là nhóm chiếm doanh thu chính. Cơ cấu sản phẩm có xu hướng chuyển dịch sang nhóm

*Trường Đại học Dược Hà Nội

Chịu trách nhiệm chính: Nguyễn Thị Song Hà

Email: songhaktd@gmail.com

Ngày nhận bài: 8.3.2021

Ngày phản biện khoa học: 27.4.2021

Ngày duyệt băi: 11.5.2021 sản phẩm TPBVSK và nhóm sản phẩm Mỹ phẩm. Tư khóa: Cơ cấu sản phẩm, Công ty cổ phân Dược khoa.

\section{SUMMARY \\ SURVEY ON THE STRUCTURE OF PRODUCT PORTFOLIO OF DUOC KHOA JOINT STOCK COMPANY IN THE PERIOD 2015-2019}

Objectives: Surveying the structure of product portfolio of Duoc Khoa Joint Stock Company in the period 2015-2019. Subjects: Product portfolio and business performance of Duoc Khoa Joint Stock Company from 2015 to the end of 2019. Methods: Cross-sectional descriptive study. Results: The Company's product portfolio in the period of 20152019 included 4 product groups: Drugs, Dietary Supplements, Cosmetics and Medical Equipment. The group of Dietary Supplements always accounted for the highest number of products with more than $50 \%$ of products annually. The group of Drugs possessed the second highest number of products with about $20 \%$ of annual products, however, it accounted for the main revenue with more than $40 \%$ of total revenue. The Cosmetics and Medical equipment group experienced a strong growth in the number of products and sales in the period of 2015- 2019. Conclusion: Drugs account for the main revenue of the company. The product portfolio structure tends to shift to Dietary Supplements and Cosmetics. 